\title{
Niveles de dolor y tolerancia a la biopsia endometrial, con ibuprofeno.
}

\author{
PALOMINO BALDEON Juan Carlos, MATTOS VILLENA Gianina, LAZO ESCALANTE Marcela, DIAZ \\ HERRERA Jorge Antonio*, QUISPE CUBA Juan Carlos**.
}

\section{SUMMARY}

Objective: To compare the levels of pain and tolerance to the endometrial biopsy with the Novak's cureta (Rigid), in patients who receive Ibuprofen $800 \mathrm{mg}$ versus placebo, 30 to $45 \mathrm{~min}$. before the procedure. Material and Methods: This is a double blind, randomized, comparative clinical trial. We included 25 patients per treatment group, one group received two capsules of Ibuprofen of 400mg PO and the other one, two capsules of placebo PO according to the sequence. Both groups were compared according to filial data, gynecological and obstetric history, the reason for the endometrial biopsy and finally the valuation of his pain experience and tolerance to the procedure. Results: The populations are comparable to each other. When evaluating the variable pain, there was statistically significant difference between values of pain in group that received Ibuprofen (Median 45mm) and values in group that received placebo (Median $65 \mathrm{~mm}$ ) with p=0.0137.

There was not statistically significant difference in the variable tolerance between group that received Ibuprofen (Median $37 \mathrm{~mm}$ ) and group that received placebo (Median $53 \mathrm{~mm}$ ) with $\mathbf{p}=\mathbf{0 . 2 3}$. Conclusion: We conclude that the use of $800 \mathrm{mg}$ of oral Ibuprofen 30 to 45 minutes before the endometrial biopsy in patients older than 44 years diminishes their pain experience during the procedure. ( Rev Med Hered 2003; 14: 122-127).

KEY WORDS: Pain, tolerance, ibuprofen, endometrial biopsy.

\section{RESUMEN}

Objetivo: Comparar los niveles de dolor y tolerancia a la biopsia endometrial con la cureta de Novak (Rígida), en pacientes que reciben Ibuprofeno 800mg vs placebo, 30 a $45 \mathrm{~min}$. antes del procedimiento. Material y Métodos: Se realizó un estudio ensayo clínico, doble ciego, randomizado y comparativo. Se incluyerón 25 pacientes por grupo de tratamiento quienes recibieron dos cápsulas de Ibuprofeno de 400mg VO o dos cápsulas de placebo VO según corresponda. Se comparó entre ambos grupos, datos de filiación, antecedentes gineco-obstétricos, motivo de la biopsia de endometrio y finalmente valoración de su experiencia de dolor y tolerancia al procedimiento. Resultados: En poblaciones comparables al evaluar la variable dolor se encuentra que existe diferencia estadísticamente significativa entre los valores de dolor del grupo que recibió Ibuprofeno (Mediana 45mm) contra el grupo que recibió placebo (Mediana $65 \mathrm{~mm}$ ) con un $\mathrm{p}=0.0137$. No se encuentra diferencia en cuento a la valoración de la tolerancia entre el grupo que recibió Ibuprofeno (Mediana $37 \mathrm{~mm}$ ) contra el grupo que recibió placebo (53mm) con un $\mathrm{p}=0.23$. Conclusión: Se concluye que la utilización de $800 \mathrm{mg}$ de Ibuprofeno vía oral 30 a 45 minutos antes de la biopsia endometrial en pacientes mayores de 44 años disminuye su experiencia de dolor durante el procedimiento. ( Rev Med Hered 2003; 14: 122-127).

PALABRAS CLAVE: Dolor, tolerancia, ibuprofeno, biopsia de endometrio.

\footnotetext{
* Médico Gineco-Obstetra. Jefe del Dpto. de Gineco-Obstetricia del Hospital Nacional Cayetano Heredia. Jefe del Dpto Académico de Gineco-obstetricia de la UPCH.

** Médico Gineco-Obstetra. Médico Asistente, Profesor Auxiliar del Dpto. de Gineco-Obstetricia de la UPCH.
} 


\section{INTRODUCCION}

En 1925, el ginecólogo Howard Kelly (1) describe un procedimiento en lugar de la Dilatación y Curetaje que puede ser realizado sin anestesia en el consultorio ambulatorio. Posteriormente en 1935 Novak introduce una nueva cureta delgada la cual puede ser introducida más fácilmente en la cavidad uterina sin necesidad de dilatación cervical (1). En los últimos años los dispositivos flexibles descartables (Pipelle, Explora, Zsampler) son los de mayor uso a nivel mundial $(2,3)$. Sin embargo en países en vías de desarrollo como el nuestro todavía se utilizan los dispositivos rígidos, principalmente por motivos económicos.

En el caso de los dispositivos flexibles(Pipelle y/o Explora), el principio básico es la creación de una presión negativa al retirar el embolo de dentro de la vaina, estando dentro de la cavidad uterina. En el caso de las curetas rígidas (Novak y/o Randolph) se realiza un curetage del tejido endometrial (4).

El efecto indeseable más importante de la biopsia endometrial es el dolor, que es de mayor intensidad con los dispositivos rigidos (5).

Se recomienda la premedicación con un analgésico no-esteroideo oral que puede ser utilizado 30-45 minutos antes del procedimiento (eg, Ibuprofeno 600$800 \mathrm{mg})(1,5,6)$.

El objetivo del presente trabajo fue comparar los niveles de dolor y tolerancia a la biopsia endometrial con la cureta de Novak (rígida) en pacientes que recibieron 30-45 min. antes del procedimiento Ibuprofeno $800 \mathrm{mg}$ vs placebo.

\section{MATERIAL Y METODOS}

Tipo de Estudio: Es un ensayo clínico, doble ciego, randomizado y comparativo. Este trabajo fue aprobado por el Comité de Etica del Hospital Nacional Cayetano Heredia.

Población: Las pacientes fueron captados en Consultorios Externos del servicio de Ginecología del Hospital Nacional Cayetano Heredia entre los meses de Marzo 2000 y Febrero 2001.

\section{Criterios de Inclusión:}

- Pacientes con indicación de Biopsia de endometrio por hallazgo en ultrasonido transvaginal de endometrio engrosado con o sin sintomatología asociada.
- Edad mayor de 44 años con o sin terapia de reemplazo hormonal.

- Relaciones sexuales positivas.

- Consentimiento para participar.

Criterios de Exclusión:

- Embarazo

- Enfermedad previa de endometrio menor a dos años, cirugía ginecológica menor a dos años, estenosis cervical, neoplasia ginecológica, dolor pélvico crónico.

- Endometritis aguda

- Usuaria de DIU.

- Historia de reacción alérgica hacia Anti-inflamatorios no esteroideos.

- Pacientes con enfermedad crónica actual (Asma bronquial, Tuberculosis, Diabetes, Artritis Reumatoide, Cáncer, Brucella, LES, Coagulopatía, úlcera péptica, insuficiencia renal crónica, enfermedad psiquiátrica, etc)

- Mayor o igual a dos intentos fallidos de Biopsia.

Las pacientes ingresaron al estudio en forma aleatoria de acuerdo a un número random, previo consentimiento informado escrito. Luego toda paciente recibió 30 a 45 minutos antes del procedimiento dos cápsulas de 400mg de ibuprofeno o dos cápsulas de placebo, las que presentaban las mismas características físicas externas, luego el autor JCPB explicaba en forma oral a cada paciente las definiciones de dolor y tolerancia(las cuales también estaban en el consentimiento informado). El residente de segundo año de Ginecología y Obstetricia de turno procedía a realizar la biopsia endometrial por la técnica habitual (4).

Variables: Se evalúo la edad, grado de instrucción, antecedentes Gineco-obstétricos(G-O) (Fecha de última regla, gestaciones, edad de primera relación sexual, fecha de última relación sexual, número de compañeros sexuales, enfermedad de transmisión sexual, utilización de métodos anticonceptivos, presencia de dispareunia, presencia de dismenorrea), motivo de biopsia endometrial, Score de Dolor, Score de Tolerancia e histerometría.

Medida del Dolor y Tolerancia: Se realizó con la escala visual análoga (EVA). En resumen, son dos líneas dibujadas en la ficha de 100 (Cien) mm de longitud, donde la paciente es preguntada por su experiencia de dolor y tolerancia al procedimiento $(0=$ no hay dolor $\mathrm{y}$ $100=$ el peor dolor $0=$ muy tolerable y $100=$ completamente intolerable respectivamente). El puntaje será la medida de la distancia entre la línea marcada por la paciente y el punto cero. 
Análisis Estadístico:

Se realizó un piloto de 10 pacientes por grupo de tratamiento, para hallar el numero de muestra según la fórmula que corresponde a la comparación de porcentajes de una variable determinada (dolor) entre poblaciones (7)

$$
N=\frac{(Z \alpha+Z \beta)^{2}\left(p 1 q 1+p^{2} q^{2}\right)}{(p 1-p 2)^{2}}
$$

p1 : \% de pac. del grupo de tx $\mathrm{N}^{\circ} 1$ que presentan dolor $>50 \mathrm{~mm}$.

p2 : \% de pac. del grupo de tx $\mathrm{N}^{\circ} 2$ que presentan dolor $>50 \mathrm{~mm}$.

q1: $\%$ de pac. del grupo de tx $\mathrm{N}^{\circ} 1$ que presentan dolor $<50 \mathrm{~mm}$.

q2 :\% de pac. del grupo de tx $\mathrm{N}^{\circ} 2$ que presentan_dolor $<50 \mathrm{~mm}$

Z $\alpha$ : Nivel de significancia: 1,96

Zß: Potencia de la prueba: 0,90

Tx $\mathbf{N}^{\circ}$ 1: Ibuprofeno

Tx N ${ }^{\circ}$ 2: Placebo

Se halló para Ibuprofeno p1: 0.3 y para placebo p2: 0.7 . Así obteniendo que para cada grupo se necesitaba 21 pacientes. Por ser datos de distribución irregular o asimétricos se utilizó estadística no paramétrica. Se tomó como medida de tendencia central a la mediana y como medida de dispersión a los percentiles para las escalas de dolor y tolerancia. Usando para su respectivo análisis estadístico la prueba U de Mann Whitney. Para las variables numéricas se utilizó la t de student y para las variables categóricas se utilizó la prueba de $\mathrm{Chi}^{2}$. Se utilizó como paquete estadístico el programa SPSS Décima Versión.

\section{RESULTADOS}

Se incluyeron 25 pacientes por grupo. La indicación de la biopsia endometrial en ambos grupos fue por hallazgo ecográfico de endometrio engrosado examen realizado por presentar sangrado anormal tanto en el grupo Ibuprofeno (60\%) y el de Placebo (52\%)(p>0.05).

No hubo diferencias estadísticamente significativas entre grupos con respecto a datos de filiación y antecedentes $\mathrm{G}-\mathrm{O}\left(\right.$ Cuadro $\mathrm{N}^{\mathrm{o}} 1$ y $\mathrm{N}^{\mathrm{o}} 2$ ).

Se encontró diferencia entre las medianas de los puntajes de dolor para ibuprofeno 45mm (Q1-Q3: 2060) y placebo $65 \mathrm{~mm}$ (Q1-Q3:41-89) con un $\mathrm{p}=0.0137$. Por otro lado, aunque hay diferencia con el escore de tolerancia para Ibuprofeno 37mm(Q1-Q3: 29-48) contra placebo $53 \mathrm{~mm}(\mathrm{Q} 1-\mathrm{Q} 3: 26-80)$ esta no llega a ser estadísticamente significativa $(\mathrm{p}=0.23)$. (Cuadro $\left.\mathrm{N}^{\circ} 3\right)$.

Con la finalidad de clasificar el dolor según su intensidad, se dividió en tres partes la longitud de la escala visual análoga, clasificándose en dolor leve (Segmento más cercano al cero(0)), dolor moderado (Segmento intermedio) y dolor severo (Segmento más cercano al diez(10)); encontrando que para Ibuprofeno se tuvo ocho( $32 \%)$, quince $(60 \%)$ y dos $(8 \%)$ pacientes; mientras que para placebo cuatro (16\%), once (44\%) y diez (40\%) pacientes presentando dolor leve, moderado y severo respectivamente.

\section{DISCUSION}

Las poblaciones en estudio son comparables entre ellos, no existiendo diferencias estadísticamente significativas entre las variables de filiación ni antecedentes ginecoobstétricos.

La complicación más frecuente de la biopsia endometrial es el dolor producido por los cólicos uterinos el cual difiere de acuerdo al dispositivo usado $(5,6)$. Otras complicaciones pueden ser perforación uterina, reacción vasovagal pero son realmente infrecuentes (4).

Los dispositivos rígidos producen mayores niveles de dolor que aquellos flexibles; por ejemplo en mujeres infértiles, el $13 \%$ en las que se utilizó Pipelle

Cuadro No1. Datos de Filiación Según Droga Recibida.

\begin{tabular}{|c|c|c|c|c|c|}
\hline \multirow[b]{2}{*}{ INSTRUC.* } & \multicolumn{2}{|c|}{ Ibuprofeno } & \multicolumn{2}{|c|}{ Placebo } & \multirow[t]{2}{*}{$\mathbf{P}$} \\
\hline & $\mathrm{n}$ & $\%$ & $\mathrm{n}$ & $\%$ & \\
\hline Analfabeta & 2 & $8.00 \%$ & 1 & $4.00 \%$ & $>0.05$ \\
\hline Primaria & 2 & $48.00 \%$ & 11 & $44.00 \%$ & $>0.05$ \\
\hline Secundaria & 8 & $32.00 \%$ & 10 & $40.00 \%$ & $>0.05$ \\
\hline Superior & 3 & $12.00 \%$ & 3 & $12.00 \%$ & $>0.05$ \\
\hline EDAD+ & \multicolumn{2}{|c|}{$51.04+7.05(\mathrm{x}+\mathrm{s})$} & \multicolumn{2}{|c|}{$48.2+4.14(\mathrm{x}+\mathrm{s})$} & $>0.05$ \\
\hline
\end{tabular}

*Usando prueba de $\mathrm{X}^{2}$

+Usando prueba de U de Mann-Whitney 
Cuadro No2. Antecedentes Gineco-Osbtetricos Según Grupo de Estudio

\begin{tabular}{|l|c|c|c|c|c|}
\hline & \multicolumn{2}{|c|}{ Ibuprofeno } & \multicolumn{2}{|c|}{ Placebo } & P \\
\hline & $\mathrm{n}$ & $\%$ & $\mathrm{n}$ & $\%$ & \\
\hline FUR & 7 & $28.00 \%$ & 8 & $32.00 \%$ & \\
$>6$ MESES & 18 & $72.00 \%$ & 17 & $68.00 \%$ & $>0.05$ \\
$<6$ MESES & 22 & $88.00 \%$ & 22 & $88.00 \%$ & \\
ETS & 3 & $12.00 \%$ & 3 & $12.00 \%$ & n.a \\
No & 16 & $64.00 \%$ & 15 & $60.00 \%$ & \\
Si & 9 & $36.00 \%$ & 10 & $40.00 \%$ & $>0.05$ \\
URS & 14 & $56.00 \%$ & 16 & $64.00 \%$ & \\
$<3$ meses & 11 & $44.00 \%$ & 9 & $36.00 \%$ & $>0.05$ \\
$>3$ meses & 16 & $64.00 \%$ & 17 & $68.00 \%$ & \\
DISMENORREA & 9 & $36.00 \%$ & 8 & $32.00 \%$ & $>0.05$ \\
No & & & & & \\
Si & 14 & $56.00 \%$ & 12 & $48.00 \%$ & $>0.05$ \\
DISPAREUNIA & 11 & $44.00 \%$ & 13 & $48.00 \%$ & \\
No & & & & \\
Si & & & & \\
MAC & & & & \\
No & & & & \\
Si & & & & & \\
\hline
\end{tabular}

n.a no aplicable

Usando prueba de $\mathrm{X}^{2}$

n.a no aplicable

Usando prueba de $\mathrm{X}^{2}$

FUR: Fecha de última regla.

ETS: Enfermedad de transmisión sexual.

URS: Ultima relación sexual.

MAC: Uso de Métodos anticonceptivos.

presentaron un dolor intenso, en comparación con la cureta Novak que produjo en un $60 \%$ de los pacientes dolor severo (8). Silver encuentra en mujeres con cáncer de endometrio, en las que se utiliza la cureta Pipelle, dolor leve hasta en un $80 \%$, dolor moderado en un $15 \%$ $\mathrm{y}$ dolor severo en un 5\% (9). Stovall encuentra en mujeres con hemorragia uterina anormal dolor severo en $17 \%$ utilizando la cureta Novak contra $6.7 \%$ utilizando la cureta Pipelle (10).

Nosotros encontramos dolor severo en un $40 \%$ de las pacientes del grupo Placebo y $8 \%$ en el de Ibuprofeno.

En cuanto a tolerancia existe diferencia entre ambas poblaciones a favor del grupo de ibuprofeno, es decir presenta mayor tolerancia al procedimiento, sin embargo, esta diferencia no llega a ser estadísticamente significativa. Durante la realización del estudio, se apreció que el concepto de "tolerancia" por las pacientes era más difícilmente entendido que el de dolor. El factor que podría haber influenciado en la comprensión sería el grado de instrucción de las pacientes, porque mas del $40 \%$ de ellas solo tenían estudios primarios.

Por otro lado los estudios en los que se evalúa la tolerancia, como por ejemplo la tolerancia al tratamiento analgésico con Ibuprofeno en diferentes patologías que producen dolor, la evaluación es de tipo seguimiento por lo que dura por lo menos 10 días de duración $(11,12)$ lo que si habría influenciando en nuestros resultados.

Cuando se evalúa la sensación de dolor para ambos grupos existe una diferencia a favor del grupo de ibuprofeno $(\mathrm{p}<0.05)$, es decir la premedicación con Ibuprofeno estaría disminuyendo la sensación de dolor de las pacientes en $20 \mathrm{~mm}$ según la EVA.

El dolor es enteramente un fenómeno subjetivo y que puede ser medido solamente por el paciente (13). Hay una variedad de herramientas de valoración del dolor que muestran ser válidos y confiables (14), sin tener en cuenta la naturaleza u origen del dolor (15). Un método validado popular (16) utilizado para la medición del dolor es la escala visual análoga (EVA) $(17,18)$.

Ibuprofeno es un agente antiinflamatorio no esteroideo (AINE) que se utiliza para el alivio de dolor agudo y crónico (19). Además es efectivo como monodosis en el alivio de dolor agudo (20). Ibuprofeno se utiliza para el alivio de cefaleas, síndromes de dolor 


\section{Cuadro N³ Comparación de Medianas del Score de \\ Dolor y Tolerancia Según Grupo de Estudio}

\begin{tabular}{|l|c|c|c|c|}
\hline Variable & Mediana & $\mathbf{Q 1 - Q 3}$ & $\mathbf{Z}$ & $\mathbf{p}$ \\
\hline $\begin{array}{c}\text { Score dolor } \\
\text { Ibuprofeno }\end{array}$ & 45 & $20-60$ & -2.465 & \\
Placebo & 65 & $41-89$ & & $0.0137^{* *}$ \\
\hline $\begin{array}{c}\text { Score tolerancia } \\
\text { Ibuprofeno }\end{array}$ & 37 & $29-48$ & -1.2034 & \\
$\quad$ Placebo & 53 & $26-80$ & & 0.23 \\
\hline
\end{tabular}

$* * \mathrm{P}<0.01$

Usando prueba de U de Mann-Whitney musculoesquelético, artritis inflamatoria (21) y como tratamiento para el síndrome premenstrual y la dismenorrea primaria (22). Se sugiere utilizarlo como premedicación analgésica en la biopsia de endometrio $(2,5,6)$ sin embargo, estas afirmaciones no tienen sustento científico sólido (23); están basadas en recomendaciones de expertos o de experiencia clínica.

Los efectos adversos mas frecuentes de los AINE son a nivel gastrointestinal que pueden presentarse desde un $3 \%$ hasta $37 \%(24,25)$, otros efectos menos frecuentes son daño renal reversible y desórdenes electrolíticos (26). Sin embargo, la evaluación entre antiinflamatorios no esteroideos no selectivos, Ibuprofeno (Dosis 1200mg) tiene el menor riesgo relativo de producir molestias gastrointestinales (27) aunque este riesgo relativo bajo, no se puede asumir cuando la dosis se incrementa a mas de $1600 \mathrm{mg} / \mathrm{dia}$ (28). La mayoría de estudios que evalúan la toxicidad y efectos adversos de ibuprofeno están basados en tratamientos multidosis $>800 \mathrm{mg} /$ dia y una duración de por lo menos 3 meses. Es decir no se encuentra en la literatura el riesgo real de producir lesión gastrointestinal-de lejos la más frecuente e importantecon el uso de $800 \mathrm{mg}$ VO dosis única. Sin embargo, se puede inferir que este debe de ser mínimo, si bien es cierto no es motivo de este trabajo, en nuestras pacientes post procedimiento, ninguna paciente comunicó algún efecto adverso importante.

Finalmente concluimos que Ibuprofeno $800 \mathrm{mg}$ vía oral 30-45min antes de la biopsia endometrial en mujeres mayores de 44 años y sin patología de fondo, disminuye el dolor producido durante la biopsia endometrial utilizando la cureta rígida de Novak. Sin embargo, no es significativo el cambio de la Tolerancia al procedimiento, aunque esto podría deberse por la metodología de evaluación de la variable.

Este hallazgo nos lleva a recomendar con fundamento científico el uso de esta medicación cuando utilicemos, preferentemente, una cureta rígida al realizar la biopsia endometrial, como ocurre en la mayoría de centros de atención ginecológicos.

\section{Correspondencia:}

Juan Carlos Palomino Baldeón

Calle $2 \mathrm{~N}^{\circ} 595$ Dpto. 302 Monterrico Norte

San Borja Lima Perú

\section{REFERENCIAS BIBLIOGRAFICAS}

1. Lurain J. Diagnóstico: Biopsia Endometrial. En Berek JS, Hillard PA, Adashi EY editores Ginecología de Novak. $12^{\circ}$ Ed. California McGraw-Hill 1998;pp 1063.

2. Thomas J. Endometrial Biopsy. Am Fam Physician 2001; 63:1131-41

3. Chambers J, Chambers S. Endometrial sampling: When? Where? why? With what? Clin Obst Gynecol 1992; 35:2839.

4. Pfenninger. Procedures for Primary Care Physicians. Endometrial Biopsy. Mosby-Year Book.1994 ;pp 563-570.

5. Shelly M. Endometrial biopsy. AM Fam Physician;1997;. 55:1731-6.

6. Apgar S, Newkirk R. Endometrial Biopsy. Primare Care; Clin Offic Pract 1997;24:303-326.

7. Mormontoy W. Elaboración de Protocolos de Investigación, en Ciencias de la Salud, de la Conducta y Areas Afines. Diseño Estadístico del muestreo.1995; pp:54-5

8. Check J, Chase J, Nowroozi K, et al. Clinical evaluation of the pipelle endometrial curette for timed endometrial biopsies. J Reprod Med 1989; 34:218-220. 
9. Silver M, Miles P. Comparison of Novak and Pipelle endometrial biopsy. Obstet Gynecol 1991; 78:828-830.

10.Stovall T, Ling F, Morgan P.A prospective,randomized comparison of the Pipelle endometrial sampling device with the Novak curette.Am J Obstet Gynecol.1991;65 : 1287-90.

11.Day R, Morrison B, Luza A, et al. A randomized trial of the efficacy and tolerability of the COX-2 inhibitor rofecoxib vs ibuprofen in patients with osteoarthritis. Rofecoxib/Ibuprofen Comparator Study Group. Arch Intern Med 2000;160(12):1781-1787.

12.Hosie J, Distel M, Bluhmki E. Efficacy and Tolerability of Meloxican versus Piroxicam in Patients with Osteoarthritis of the Hip or Knee:A six- Month DobleBind Study.Clin Drug Invest 1997;13:175-184.

13.Koopman: Measurement Techniques for several Variables of Importance in Outcome Assessment: PAIN En: Arthritis and Allied Conditions, $13^{\text {th }}$ ed.Williams \& Wilkins 1997;p:6.

14.Abrahm J, Snyder J. Pain assessment and management. Clin in Office Practice 2001;28:232-38.

15.Katz, Melzack. Measurement of Pain. Surg Clin of Nort Am 1999;79:231-259.

16.Price D, McGrath P, Rafii A, et al. The validation of visual analogue scales as ratio scale measures for chronic and experimental pain. Pain 1983;17(1):45-56.

17.Scherder E, Bouma A. A Visual analogue scales for pain assessment in Alzheimer's disease.Geront 2000;46(1):4753.

18.Soyannwo O, Amanor-Boadu S, Sanya A. Pain Assessment in Nigerians-Visual Analogue Scale and Verbal Rating Scale compared.West Afr J Med 2000;19:242-5.

19.Barkin D, Pharmacologic Management of Acute and
Chronic Pain: Focus on Drug Interactions and PatientSpecific Pharmacotherapeutic Selection .South Med J 2001;94:756-812.

20.Collins S, Moore R, McQuay H et al. Edwards JE. Single dose oral ibuprofen and diclofenac for postoperative pain (Cochrane Review). In: The Cochrane Library,2001, Issue 2.

21.Donald D. Adverse reactions to nonsteroidal anti inflammatory drugs. Im Allerg Clin of North Am 1998; 18:773-798.

22.Zhang WY, Li Wan Po A Naproxen and ibuprofen provide the best pain relief for primary dysmenorrhea Evidencebased Obstetrics \& Gynecology 1999; 1:108.

23.Sackett D, Rosenberg W, Muir Gray J et al. Evidence based medicine: what it is and what it isn't. It's about integrating individual clinical expertise and the best external evidence. BMJ 1996;312:71-2.

24.Haija, A, Egbunike,I, Osemene N et al. Evaluation of the prescribing patterns and economic impact of the prevention and management of non-steriodal antiinflammatory associated gastrointestinal adverse events. ASHP Midyear Clin Meet 1999;34:P-417E

25.Lichtenstein D, Syngal S, Wolfe M. Nonsteroidal antiinflammatory drugs and the gastrointestinal tract. Arthritis Rheum 1995;38:5-18.

26.Simon LS. Nonsteroidal antiinflammatory drugs and their effects: The importance of COX selectivity. J Clin Rheumatol 1996;2:135-140.

27.Russell R. Defining patients at risk of non-steroidal anti.inflammatory drug gastropathy. Ital J Gastroenterol Hepatol 1999; 31:Supp 1: S14-18.

28.Henry, D;Lim L, García Rodríguez L et al. Variability in risk of gastrointestinal complications with individual nonsteroidal anti-inflammatory drugs:results of a collaborative meta-analysis. BMJ 1996;312:1563-1566. 